\title{
Factores asociados a las actitudes y prácticas preventivas frente a la pandemia del COVID-19
}

\author{
Irma Yupari-Azabache ${ }^{\square(0)}$ Ph.D; Jorge Díaz-Ortega ${ }^{凶(0)}$ Ph.D; \\ Ángela Rodríguez-Diaz $2,3 \bowtie$ M.SC; Ana Peralta-Iparraguirre ${ }^{凶}$ Ph.D.
}

\begin{abstract}
${ }^{1}$ Universidad César Vallejo- Instituto de Investigación, Trujillo, Perú
2Universidad César Vallejo - Facultad de Ciencias de la Salud, Trujillo, Perú.

${ }^{3}$ Centro de Salud San Martin De Porres- Medico Jefe y Responsable de Epidemiologia de Microred, Trujillo, Perú.

Correspondencia: iyupari@ucv.edu.pe
\end{abstract}

Recibido: Junio 2020; Aceptado: Octubre 2020; Publicado: Noviembre 2020.

\section{RESUMEN}

Objetivo: La presente investigación tuvo como objetivo analizar si existe relación entre los factores biológicos, sociales y culturales con las actitudes y prácticas preventivas frente a la pandemia del COVID-19 en ciudadanos de Trujillo- Perú. Material y Métodos: Tipo de estudio descriptivo, de enfoque cuantitativo y diseño correlacional, prospectivo, de corte transversal. Mediante un cuestionario previamente validado se tomaron datos de ciertos factores biológicos, sociales y culturales, así como de las actitudes y prácticas preventivas frente al COVID-19 en 185 ciudadanos de Trujillo. Por encontrarnos en cuarentena debido a la emergencia sanitaria, la recolección de datos se realizó de manera virtual. Resultados: Los resultados muestran que ciudadanos de 18 a 29 años tienen actitudes inadecuas $(12.4 \%)$, con prácticas preventivas inadecuadas $(8.6 \%)$ y también que ser de sexo masculino constituye un riesgo para tener actitud y practica inadecuada. También existen ciudadanos que son solteros o convivientes, no tienen hijos y tienen actitudes inadecuadas (14.1\%) y prácticas inadecuadas (13\%). Conclusiónes: De los factores biológicos la edad y el sexo se asocian a las actitudes y prácticas preventivas frente a la pandemia, de los sociales, el estado civil y la tenencia de hijos se asocian a las actitudes y prácticas preventivas frente a la pandemia, y del cultural el tener conocimiento de la enfermedad y de las acciones a tomar se asocia con las prácticas preventivas frente a la pandemia del COVID-19.

Palabras clave: Pandemia; COVID-19; actitudes; prácticas (Fuente: MeSH).

\section{ABSTRACT}

Objective: This research aims to analyze the relation between biological, social and cultural factors regarding the attitudes and preventive measures against COVID-19 among citizens of TrujilloPeru. Material and Methods: This is a descriptive research, with a quantitative approach and a correlational, prospective and design. A previously validated questionnaire was used to collect certain 
biological, social and cultural data, as well as attitudes and preventive measures against COVID-19 among 185 citizens of Trujillo. Due to the quarantine and the Sanitary National Emergency, the collection of data was done by virtual means. Results: Citizens between 18 and 29 years old have inappropriate attitudes (12.4\%) and inappropriate preventive measures (8.6\%). Also, males have inappropriate attitudes and inappropriate preventive measures. Moreover, single citizens or unmarried couples without children have inappropriate attitudes (14.1\%) and inappropriate practices (13\%). Conclusions: Among biological factors, age and gender are related to attitudes and preventive measures regarding the pandemic. Among social factors, marital status and parenthood are related to attitudes and preventive measures regarding the pandemic. And among the cultural factor, knowledge of this disease and the measures to be taken are related to the preventive measures against COVID-19.

Keywords: Pandemic; COVID-19; attitudes; practices (Source: MeSH)

\section{INTRODUCCION}

Desde que se conocieron los primeros casos de neumonía en pobladores de Wuhan provocado por SARS-CoV2, probablemente ocasionado por una zoonosis, esta se ha extendido a países de todos los continentes entre febrero y marzo, siendo últimamente España e Italia los más afectados en Europa, Estados Unidos en América del Norte y Brasil en América del Sur, sumado a las malas decisiones políticas para enfrentar a la pandemia (1).

La población es generalmente susceptible a este virus. Los casos de muerte son vistos con mayor frecuencia en adultos mayores $y$ aquellos con enfermedades crónicas como diabetes, hipertensión, obesidad, enfermedades cardiovasculares, aunque en la mayoría de los niños los síntomas son relativamente leves $(2,3)$. El número promedio de casos nuevos por infectado oscila entre 2.24 (IC 95\%: 1.96 2.55) y 3,58 (IC 95\%: 2.89-4.39), es decir, que una persona puede llegar a contagiar aproximadamente de 2 a 4 personas (4).

Por otro lado, una de las características del Coronavirus es su permanencia en diferentes tipos de materiales durante 2 horas hasta 9 días, permitiendo una alta probabilidad infecciosa para aquellas personas que establecen contacto con ellos (5). Así también cualquier contacto que haya proporcionado atención a un caso confirmado mientras el caso presentaba síntomas, constituye un riesgo de contagio (6).

Desinfectantes como formaldehído, peróxido de hidrógeno y alcohol, han sido seleccionados (7), así como el uso de hipoclorito de sodio a una concentración de $0.1 \%$ es efectivo en $1 \mathrm{~min}$ sobre el virus. La OMS también recomienda una concentración de etanol al 70\% para desinfectar superficies pequeñas (5).

En América Latina, el escenario es complicado ya que existen otras enfermedades infecciosas epidémicas que causan fiebre, en la que los sistemas de salud están comprometidos, como es el caso del dengue y sarampión. La higiene respiratoria debe ser parte de los rituales y obligatoria a menudo, sin embargo, el hacinamiento y diversas interacciones sociales comunes normalmente es ahora un riesgo (8). A la fecha no hay un tratamiento específico frente al virus, y únicamente en situaciones clínicas donde el paciente puede tener riesgo de infección se utilizan antibióticos, así mismo se han probado con antivirales como lopinavir/ ritonavir, en pacientes con la fase temprana del virus (9).

Las noticias generan ansiedad y miedo en la población, fomentan rumores, así mismo personas afectadas por la enfermedad pueden estar sujetos al rechazo social y la discriminación (10).

En Perú el 06 de marzo del presente año se dio a conocer el primer caso de coronavirus y a pesar de que el gobierno declarara al país en estado de emergencia a partir del 16 de marzo, la situación es preocupante dado que los casos diariamente han ido en aumento y muchos hospitales en diferentes departamentos del país han colapsado, al 21 de mayo ya pasamos los 100000 casos de personas infectadas, con una letalidad de $2.91 \%$. El departamento de La Libertad es uno de los más afectados acercándonos a 2500 contagiados y con una letalidad de $3.8 \%$, siendo el distrito de Trujillo el que presenta la mayor cantidad de casos y muchas personas del personal de salud, policías y fuerzas armadas se han contagiado $(11,12)$. 
Por todo ello nos hemos permitido el desarrollo del presente estudio de investigación y desde nuestro entorno social y cultural nos planteamos la pregunta de investigación ¿Existe asociación entre los factores biológicos, sociales y culturales con las actitudes y prácticas preventivas frente a la pandemia del COVID-19 en los ciudadanos el distrito de Trujillo? Se consideró como hipótesis de investigación que si existe asociación entre los factores biológicos, sociales y culturales con las actitudes y prácticas preventivas frente a la pandemia del COVID-19 en ciudadanos del distrito de Trujillo. El objetivo general de esta investigación es analizar si existe relación entre los factores biológicos, sociales y culturales con las actitudes y prácticas preventivas frente a la pandemia del COVID-19, teniendo como objetivos específicos determinar si existe asociación entre los factores biológicas como edad y sexo con las actitudes y prácticas preventivas frente a la pandemia del COVID-19, determinar si existe asociación entre los factores sociales como estado civil, grado de instrucción, si tiene hijos y cuenta con trabajo estable con las actitudes y prácticas preventivas frente a la pandemia del COVID-19 y determinar si existe asociación entre el factor cultural como nivel de conocimiento y sus dimensiones con las actitudes y prácticas preventivas frente a la pandemia del COVID-19.

\section{MATERIAL Y MÉTODOS}

Tipo y diseño de estudio. Tipo de estudio descriptivo, de enfoque cuantitativo y diseño correlacional, prospectivo, de corte transversal (13).

Población de estudio. La población estuvo conformado por todos los ciudadanos del distrito de Trujillo (de 18 a 70 años), con acceso a internet; las encuestas se tomaron en el periodo del 16 de marzo al 12 de abril, de manera virtual por encontramos en periodo de aislamiento social decretado por el gobierno peruano. Los cuestionarios fueron administrados utilizando un muestreo no probabilístico por conveniencia, seleccionando una muestra de 185 ciudadanos que residen en el distrito de Trujillo, la cual fue calculada con la fórmula para población infinita, utilizándose un nivel de confianza del $95 \%$, y un error ajustado del 7\% (14).

Técnicas e instrumentos de recolección de datos. Para la recolección de datos, la técnica aplicada fue la encuesta y el instrumento un cuestionario, el cual fue validado por cinco profesionales: tres médicos cirujanos, un infectólogo y un metodólogo, obteniendo una $\mathrm{V}$ de aiken de $99 \%$ para el instrumento de conocimiento, $100 \%$ para el instrumento de actitudes y $100 \%$ para el instrumento de prácticas. Así también para medir la confiabilidad del instrumento de nivel de conocimiento, a nivel general con 34 ítems se obtuvo aplicando la fórmula de Kuder Richardson que dio un valor de 0.85 e indica una confiabilidad buena, y en sus dimensiones para conocimiento de síntomas con 10 ítems dio un valor de 0.94 con una confiabilidad elevada, para conocimiento de formas de contagio con 8 ítems dio un valor de 0.90 con una confiabilidad muy buena, para conocimiento de la enfermedad con 8 ítems dio un valor de 0.72 que indica una confiabilidad respetable y para conocimiento de acciones a tomar también con 8 ítems dio un valor de 0.76 que indica una confiabilidad muy respetable. La segunda parte evalúa actitudes preventivas hacia el COVID-19 y consistió en 9 preguntas, las cuales fueron sometidas al análisis de confiabilidad del alpha de crombach que resultó con una confiabilidad respetable al obtenerse el valor de 0.71 . Por último, en la tercera parte se midió las prácticas preventivas frente al COVID-19, la confiabilidad de estos ítems se evaluó aplicando también la fórmula de del alpha de crombach, y se obtuvo un valor de 0.78 que corresponde a una confiabilidad muy respetable $(13,15)$.

Análisis estadístico. Para el análisis de la información, se elaboró una base de datos en Excel, se realizó el análisis en el Programa SPSS versión 26. El nivel de conocimiento fue analizado a nivel general y por dimensiones. El análisis bivariado se realizó con la prueba de contrastación de hipótesis Chi cuadrado y OR para identificar si los factores biológicos, sociales y culturales se asocian y en algunos casos constituye un factor de riesgo para las actitudes y prácticas preventivas para el COVID-19 (14).

\section{RESULTADOS}

Al evaluar los factores biológicos con las actitudes y prácticas preventivas frente a la pandemia del COVID-19, podemos apreciar que la mayoría de ciudadanos encuestados tienen una edad de 30 a 59 años con actitudes y prácticas adecuadas (43.2 y $60.5 \%$ respectivamente). También apreciamos que son del sexo masculino y tienen actitudes y prácticas adecuadas ( 23.2 y $25.4 \%$ respectivamente); confirmamos una asociación significativa entre la edad y sexo con las actitudes 
y prácticas preventivas frente al COVID-19 $(p<0.05)$. Así también podemos afirmar que existe 2.61 veces mayor probabilidad de que una persona con sexo masculino tenga una actitud inadecuada y 2.74 veces mayor probabilidad de que tenga una práctica preventiva inadecuada frente a la pandemia del COVID-19 (Tabla 1)

Tabla 1. Relación entre los Factores Biológicos con las actitudes y prácticas preventivas frente a la pandemia del COVID-19 en los ciudadanos de Trujillo - La Libertad Perú. 2020.

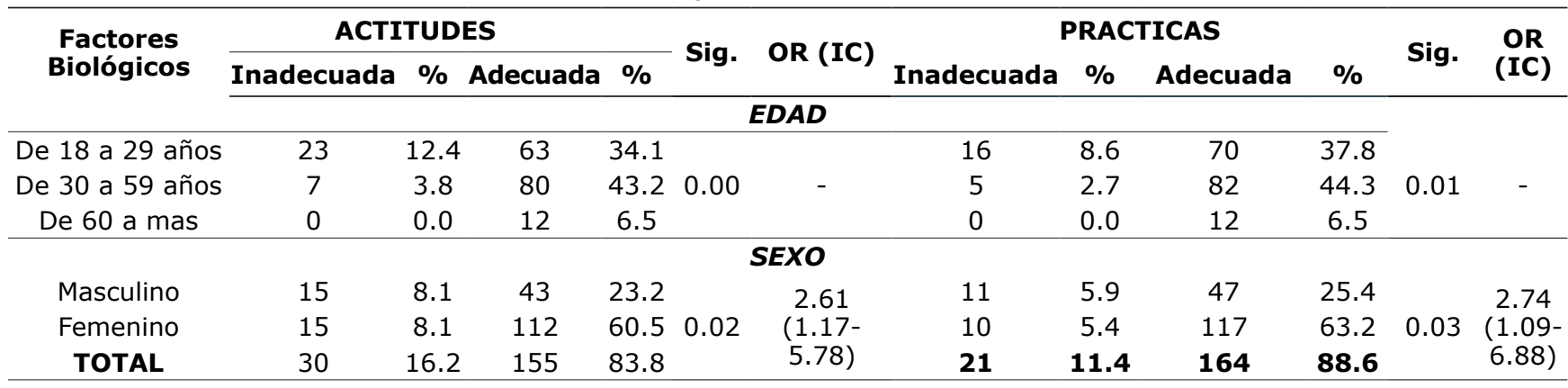

Fuente: Encuesta aplicada a los ciudadanos de Trujillo

Al analizar los factores sociales, se observó que la mayoría de ciudadanos encuestados que tienen actitudes y practicas adecuadas, son solteros o convivientes ( 48.6 y $53 \%$ respectivamente), no tienen hijos ( 42.2 y $45.4 \%$ respectivamente) , tienen estudios superiores (51.9 y $54.6 \%$ respectivamente), estabilidad laboral (52.4 y $56.8 \%$ respectivamente), sin embargo también existe un porcentaje significativo de ciudadanos que tienen actitudes y prácticas inadecuadas, son solteros o convivientes (14.1 y $18 \%$ respectivamente) y no tienen hijos (13 y $9.7 \%$ respectivamente). Por ultimo también en el análisis se puede apreciar que existe asociación entre el estado civil y el tener hijos con las actitudes y prácticas preventivas frente al COVID-19 $(p<0.05)$, así como que existe 4.69 veces mayor probabilidad de que una persona soltera tenga una actitud inadecuada y 4 veces mayor probabilidad que tenga también practicas inadecuadas, así como que una persona que no tiene hijos tiene 3.94 veces mayor probabilidad de tener actitudes inadecuadas y 5.71 veces mayor probabilidad de tener practicas preventivas inadecuadas frente a la pandemia del COVID-19. (Tabla 2).

Tabla 2. Relación entre los Factores Sociales con las actitudes y prácticas preventivas frente a la pandemia del COVID-19 en los ciudadanos de Trujillo - La Libertad Perú. 2020.

\begin{tabular}{|c|c|c|c|c|c|c|c|c|c|c|c|c|}
\hline \multirow{2}{*}{$\begin{array}{c}\text { Factores so- } \\
\text { ciales }\end{array}$} & \multicolumn{4}{|c|}{ Actitudes } & \multirow{2}{*}{ Sig. } & \multirow{2}{*}{ OR } & \multicolumn{4}{|c|}{ Practicas } & \multirow{2}{*}{ Sig. } & \multirow{2}{*}{ OR (IC) } \\
\hline & Inadecuada & $\%$ & Adecuada & $\%$ & & & Inadecuada & $\%$ & Adecuada & $\%$ & & \\
\hline \multicolumn{13}{|c|}{ Estado civil } \\
\hline \multirow{2}{*}{$\begin{array}{c}\text { Soltero o } \\
\text { convivientes } \\
\text { Casado }\end{array}$} & 26 & 14.1 & 90 & 48.6 & \multirow{2}{*}{0.00} & \multirow{2}{*}{$\begin{array}{c}4.69 \\
(1.56- \\
14.10) \\
\end{array}$} & 18 & 9.7 & 98 & 53.0 & \multirow{2}{*}{0.02} & \multirow{2}{*}{$\begin{array}{c}4(1.14- \\
14.26)\end{array}$} \\
\hline & 4 & 2.2 & 65 & 35.1 & & & 3 & 1.6 & 66 & 35.7 & & \\
\hline \multicolumn{13}{|c|}{ Tenencia de hijos } \\
\hline No tiene hijos & 24 & 13.0 & 78 & 42.2 & \multirow{2}{*}{0.00} & \multirow{2}{*}{$\begin{array}{c}3.94 \\
(1.53- \\
10.19) \\
\end{array}$} & 18 & 9,7 & 84 & 45.4 & \multirow{2}{*}{0.00} & \multirow{2}{*}{$\begin{array}{c}5.71 \\
(1.62- \\
20.15) \\
\end{array}$} \\
\hline Si tiene hijos & 6 & 3.2 & 77 & 41.6 & & & 3 & 1,6 & 80 & 43,2 & & \\
\hline \multicolumn{13}{|c|}{ Grado de instrucción } \\
\hline $\begin{array}{l}\text { Primaria o } \\
\text { secundaria }\end{array}$ & 15 & 8.1 & 59 & 31.9 & \multirow{2}{*}{0.22} & \multirow{2}{*}{$\begin{array}{c}1.63 \\
(0.74- \\
3.57)\end{array}$} & 11 & 5.9 & 63 & 34.1 & \multirow{2}{*}{0.22} & \multirow{2}{*}{$\begin{array}{c}1.76 \\
(0.71- \\
4.39)\end{array}$} \\
\hline superior & 15 & 8.1 & 96 & 51.9 & & & 10 & 5.4 & 101 & 54.6 & & \\
\hline \multicolumn{13}{|c|}{ Estabilidad Laboral } \\
\hline NO & 12 & 6.5 & 58 & 31.4 & \multirow{3}{*}{0.79} & \multirow{3}{*}{$\begin{array}{c}1.12 \\
(0.50- \\
2.48)\end{array}$} & 11 & 5.9 & 59 & 31.9 & \multirow{3}{*}{0.14} & \multirow{3}{*}{$\begin{array}{c}1.96 \\
(0.79- \\
4.89)\end{array}$} \\
\hline SI & 18 & 9.7 & 97 & 52.4 & & & 10 & 5.4 & 105 & 56.8 & & \\
\hline TOTAL & 30 & 16.2 & 155 & 83.8 & & & 21 & 11.4 & 164 & 88.6 & & \\
\hline
\end{tabular}

Fuente: Encuesta aplicada a los ciudadanos de Trujillo 
En la Tabla 3, al evaluar el factor cultural se observa que la mayoría de ciudadanos tienen un conocimiento general bueno y un nivel adecuado de actitud y práctica, pero no se evidencia asociación entre las variables conocimiento con actitudes y prácticas preventivas frente al COVID-19, sin embargo al analizar por dimensiones podemos apreciar que existe asociación significativa $(p<0.05)$ entre el hecho de tener conocimiento de la enfermedad con actitudes y prácticas preventivas y el conocimiento de acciones a tomar con las prácticas preventivas inadecuadas frente a la pandemia del COVID-19.

Tabla 3. Relación entre el Factor Cultural: Conocimiento y sus dimensiones con las actitudes y prácticas preventivas frente a la pandemia del COVID-19 en los ciudadanos de Trujillo - La Libertad Perú. 2020.

\begin{tabular}{|c|c|c|c|c|c|c|c|c|c|c|}
\hline \multirow{2}{*}{ Factor cultural ${ }^{-}$} & \multicolumn{4}{|c|}{ Actitudes } & \multirow{2}{*}{ Sig. } & \multicolumn{4}{|c|}{ Practicas } & \multirow{2}{*}{ Sig. } \\
\hline & Inadecuada & $\%$ & Adecuada & $\%$ & & Inadecuada & $\%$ & Adecuada & $\%$ & \\
\hline \multicolumn{11}{|c|}{ Conocimiento de sintomas de la enfermedad } \\
\hline Malo & 0 & 0.0 & 17 & 9.2 & & 2 & 1.1 & 15 & 8.1 & \\
\hline Regular & 15 & 8.1 & 76 & 41.1 & 0.14 & 10 & 5.4 & 81 & 43.8 & 0.99 \\
\hline Bueno & 15 & 8.10 & 62 & 33.5 & & 9 & 4.9 & 68 & 36.8 & \\
\hline \multicolumn{11}{|c|}{ Conocimiento de formas de contagio } \\
\hline Malo & 0 & 0.0 & 7 & 3.8 & & 0 & 0.0 & 7 & 3.8 & \\
\hline Regular & 28 & 15.1 & 122 & 65.9 & 0.16 & 20 & 10.8 & 130 & 70.3 & 0.21 \\
\hline Bueno & 2 & 1.1 & 26 & 14.1 & & 1 & 0.5 & 27 & 14.6 & \\
\hline \multicolumn{11}{|c|}{ Conocimiento de la enfermedad } \\
\hline Malo & 0 & 0.0 & 4 & 2.2 & & 1 & 0.5 & 3 & 1.6 & \\
\hline Regular & 12 & 6.5 & 13 & 7.0 & 0.00 & 10 & 5.4 & 15 & 8.1 & 0.00 \\
\hline Bueno & 18 & 9.7 & 138 & 74.6 & & 10 & 5.4 & 146 & 78.9 & \\
\hline \multicolumn{11}{|c|}{ Conocimiento de acciones a tomar } \\
\hline MALO & 0 & 0.0 & 1 & 0.5 & & 1 & 0.5 & 0 & 0.0 & \\
\hline Regular & 9 & 4.9 & 27 & 14.6 & 0.26 & 9 & 4.9 & 27 & 14.6 & 0.00 \\
\hline Bueno & 21 & 11.4 & 127 & 68.6 & & 0 & 0.0 & 0 & 0.0 & \\
\hline \multicolumn{11}{|c|}{ Conocimiento general } \\
\hline Regular & 6 & 3.2 & 43 & 23.2 & & 4 & 2.2 & 45 & 24.3 & \\
\hline Bueno & 24 & 13.0 & 112 & 60.5 & 0.38 & 17 & 9.2 & 119 & 64.3 & 0.41 \\
\hline TOTAL & 21 & 11.4 & 164 & 88.6 & & 21 & 11.4 & 164 & 88.6 & \\
\hline
\end{tabular}

Fuente: Encuesta aplicada a los ciudadanos de Trujillo

En la Figura 1, se muestran las prácticas preventivas frente a la pandemia del COVID-19 en los ciudadanos de Trujillo-Perú, y se puede observar que predomina lavarse frecuentemente las manos cuando se prepara los alimentos $(88 \%)$ y después de ir al baño $(87 \%)$, evitar salir de casa $(86 \%)$ y alimentarse de comida preparada en casa ( $83 \%)$. Sin embargo es importante mencionar que un $42 \%$ de personas manifestaron que nunca separan cubiertos para comer, $14 \%$ nunca limpian la suela de su zapato cuando llegan a la casa, y un $11 \%$ que no usan mascarilla al salir a la calle.

En la Figura 2 encontramos las actitudes frente a la pandemia del COVID-19 en los ciudadanos de Trujillo, y se puede observar que la mayoría de encuestados (94\%) manifestaron estar de acuerdo con el aislamiento social, considerando que es la mejor opción para no propagar el virus, se encuentran preocupados que algún integrante de la familia pueda contraer el virus (90\%), sin embargo un $7 \%$ de ciudadanos opinaron que se está exagerando, que la enfermedad pasará y un $22 \%$ cree que simplemente es una gripe fuerte con cura.

En la Figura 3 se puede apreciar que el $95.3 \%$ de pobladores refiere que el medio de comunicación que brinda mayor información sobre el COVID-19 a los ciudadanos de Trujillo es la televisión, sin embargo también existe un $1.75 \%$ de personas que no les interesa informarse por ningún medio. 


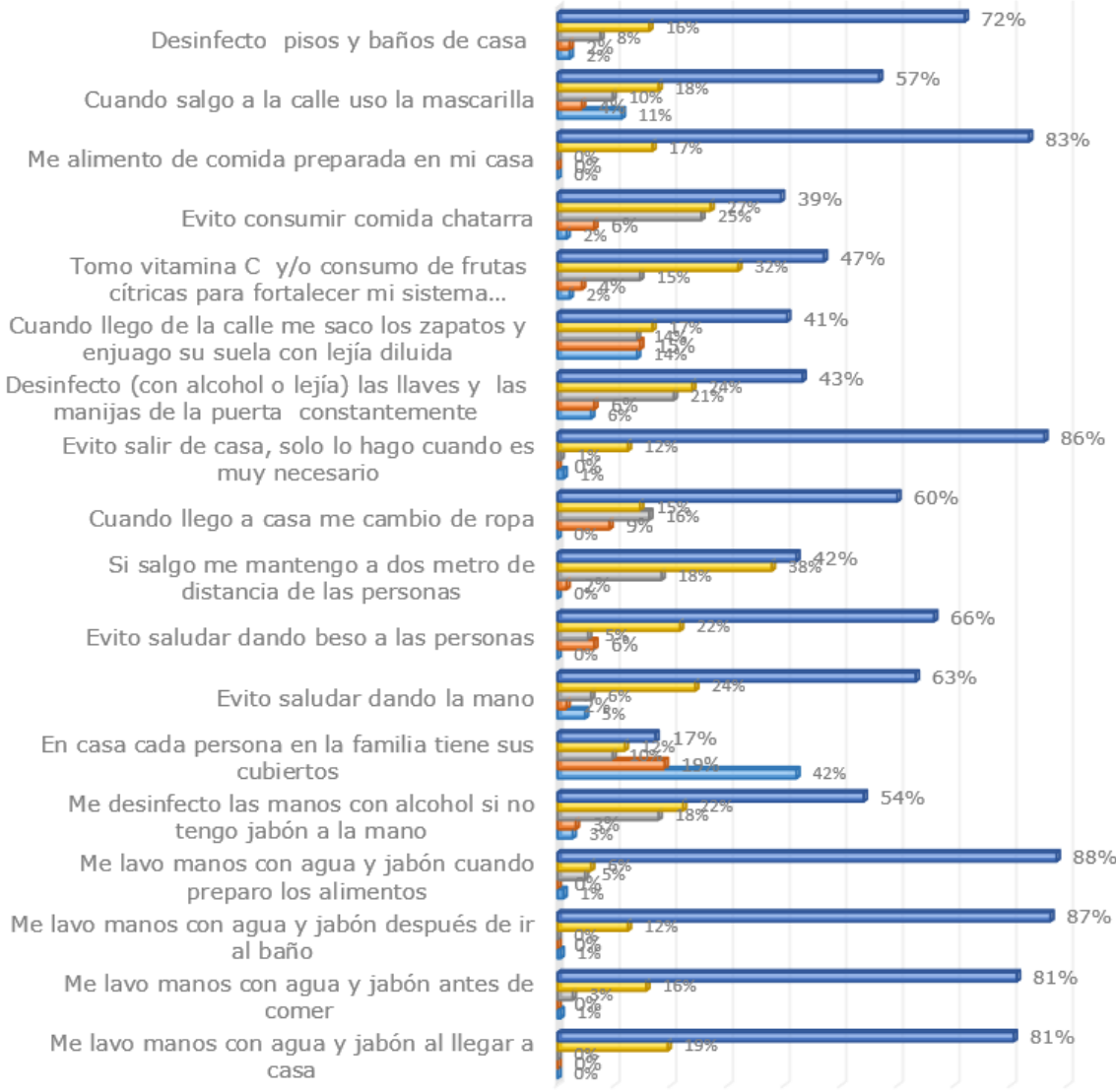

$\begin{array}{llllllllll}0 \% & 10 \% & 20 \% & 30 \% & 40 \% & 50 \% & 60 \% & 70 \% & 80 \% & 90 \%\end{array}$

Figura 1. Prácticas preventivas frente a la pandemia del COVID-19 en los ciudadanos de Trujillo - La Libertad Perú. 2020.

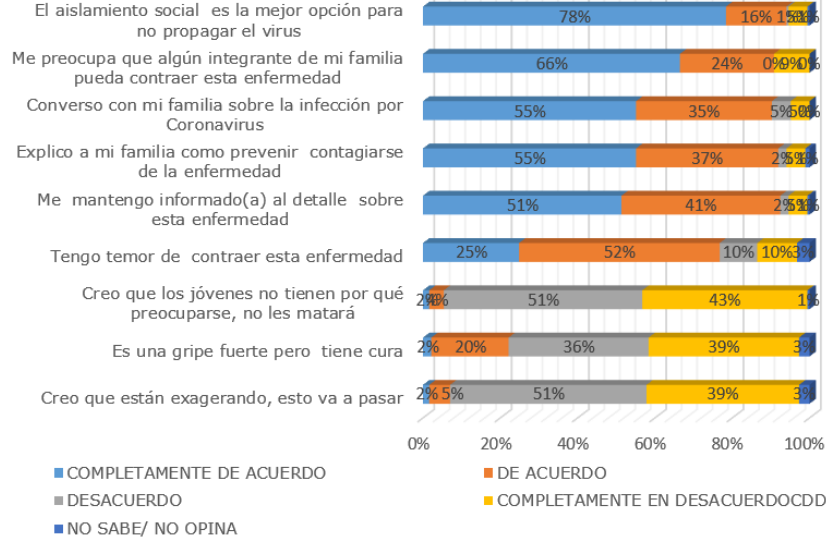

Figura 2. Actitudes frente a la pandemia del COVID-19 en los ciudadanos de Trujillo - La Libertad Perú. 2020.

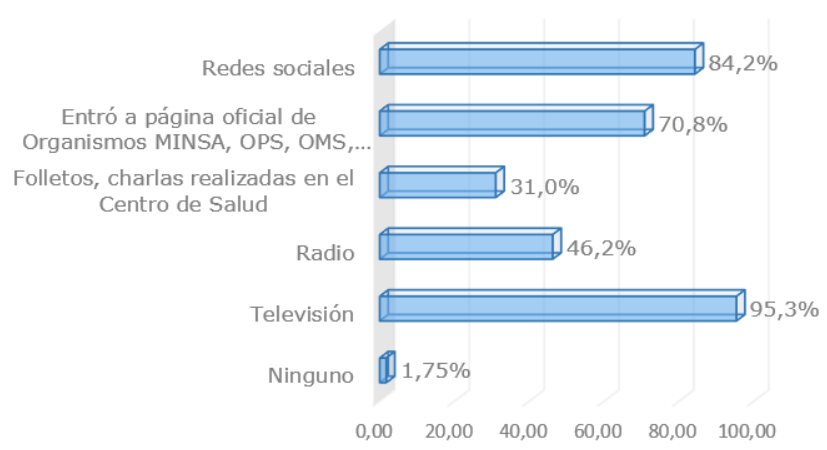

Figura 3. Medios de comunicación que brindan información sobre el COVID-19, a los ciudadanos de Trujillo - La Libertad Perú. 2020. 
Yupari-Azabache et al - Factores asociados, actitudes y prácticas preventivas del COVID-19

\section{DISCUSIÓN}

Los resultados de la Tabla 1 indican que la mayoría de ciudadanos encuestados tienen actitudes y prácticas adecuadas, sin embargo también existe un porcentaje significativo de ciudadanos con edad entre 18 a 29 años que tienen actitudes y prácticas inadecuadas (12.4 y $8.6 \%$ respectivamente), esto se asemeja con un estudio realizado en peregrinos con respecto a MERS-CoV durante la peregrinación anual del Hajj, en donde las mujeres y los adultos mayores mostraron mejor actitud y prácticas frente al MERS-CoV(16). También apreciamos que existe mayor probabilidad de que una persona de sexo masculino tenga actitudes y prácticas inadecuadas. Esto confirma los reportes mostrados por el MINSA ya que la mayor cantidad de personas contagiadas son las del sexo masculino (12), la edad avanzada y el género masculino son factores de riesgo para contraer COVID 19(17).

Los resultados de la Tabla 2 confirman la asociación entre el estado civil y el tener hijos con las actitudes y prácticas preventivas frente al COVID-19, así como que existe mayor probabilidad de que una persona soltera y que no tiene hijos tenga actitudes y prácticas inadecuadas. Por otro lado, según el análisis realizado en la Figura 1 , existe un $14 \%$ de personas que nunca limpian sus zapatos al ingresar a su casa y un $11 \%$ que no usan mascarillas al salir a la calle; en la figura 2 encontramos actitudes preocupantes como la de un $7 \%$ de ciudadanos que manifestaron que se estaba exagerando, que la enfermedad pasará y un $22 \%$ que cree que simplemente es una gripe fuerte con cura. Estas actitudes y comportamientos de la población sería una de las razones por la que los contagios se han elevado en la ciudad de Trujillo. Análogamente en un estudio similar e incluso en profesionales de salud manifestaron no hacer uso de la mascarilla en lugares donde había multitud en Arabia Saudita donde hubo reportes del Síndrome respiratorio del Medio Oriente (MERS)(18), aunque en un contexto diferente las malas prácticas pueden conducir a la propagación de la infección en la comunidad y aumentar aún más la mortalidad y la morbilidad, hecho que pudo haber sucedido en los inicios de la pandemia en nuestro país. El uso apropiado de mascarillas y respiradores es importante para proporcionar el nivel deseado de protección; sin embargo, esto también requiere conocimiento, capacitación y supervisión (19).

Los resultados observados en la Tabla 3, viéndolo de una forma positiva, se encuentran en situación similar a la de otros países como la India en donde los encuestados tenían un nivel moderado de conocimiento sobre la infección por COVID-19 y un conocimiento adecuado sobre sus aspectos preventivos, y la actitud hacia COVID-19, por ello se mostró la voluntad de las personas de seguir las pautas gubernamentales sobre cuarentena y distanciamiento social (20).

En la Figura 3 también podemos apreciar que un $1.75 \%$ de ciudadanos afirmó que no les interesa informarse de la situación por ningún medio de comunicación esto puede explicar que muchos ciudadanos no acatan las órdenes del gobierno y no han tomado conciencia del riesgo de la enfermedad por lo que se ha dado el aumento de personas contagiadas, como lo sucedido por los ciudadanos italianos que no tuvieron clara seriedad y severidad del COVID-19 y que acumuló más fatalidad que China (21).

En conclusión, podemos afirmar que de los factores biológicos la edad y el sexo se asocian a las actitudes y prácticas preventivas frente a la pandemia, resaltando que el sexo masculino es quien tiene mayor riesgo de tener prácticas preventivas inadecuadas.

De los factores sociales, el estado civil y la tenencia de hijos se asocian a las actitudes y prácticas preventivas frente a la pandemia, resaltando que existe mayor probabilidad de que una persona soltera y que no tiene hijos tenga actitudes y prácticas inadecuadas

Del factor cultural el tener conocimiento de la enfermedad y de las acciones a tomar se relaciona con las prácticas preventivas frente a la pandemia.

\section{Conflicto de intereses}

Los autores declaran que no existió conflicto de intereses de ningún tipo durante la realización y elaboración de este trabajo. 


\section{REFERENCIAS}

1. Chang $D$, Lin $M$, Wei L, Xie L, Zhu G, De la Cruz $C$, et al. Epidemiologic and Clinical Characteristics of Novel Coronavirus Infections Involving 13 Patients Outside Wuhan, China. JAMA. 2020; 323(11):10921093. http://dx.doi.org/10.1001/ jama.2020.1623

2. Jin $Y$, Cai $L$, Cheng $Z$, Cheng $H$, Deng $T$, Fan $Y$, et al. A rapid advice guideline for the diagnosis and treatment of 2019 novel coronavirus (2019-nCoV) infected pneumonia (standard version). Military Medical Research. 2020; 7(4). https:// dx.doi.org/10.1186/s40779-020-0233-6

3. Chen N, Zhou M, Dong X, Qu J, Gong $F$, Han $Y$, et al. Epidemiological and clinical characteristics of 99 cases of 2019 novel coronavirus pneumonia in Wuhan, China: a descriptive study. Lancet 2020; 395(10223):507-13. https://dx.doi. org/10.1016/S0140-6736(20)30211-7

4. Palacios $M$, Santos $E$, Velázquez $M$, León M. COVID-19, a worldwide public health emergency. Rev Clin Esp. 2020; https:// dx.doi.org/10.1016/j.rce.2020.03.001

5. Kampf G, Todt D, Pfaender S, Steinmann E. Persistence of coronaviruses on inanimate surfaces and their inactivation with biocidal agents. J Hosp Infect. 2020; 104(1):246251 https://dx.doi.org/10.1016/j. jhin.2020.01.022

6. Millán J, Rodríguez A, Camacho G, Mendoza $\mathrm{H}$, Rodríguez I, Álvarez C. A new emerging zoonotic virus of concern: the 2019 novel Coronavirus (COVID-19). Infectio. 2020; 24(3):187-192. http://dx.doi. org/10.22354/in.v24i3.848

7. Klaus J, Gnirs P, Hölterhoff S, Wirtz A, Jeglitza M, Gaber W, Gottschalk R. Disinfection of aircraft: Appropriate disinfectants and standard operating procedures for highly infectious diseases. Bundesgesundheitsblatt Gesundheitsforschung Gesundheitsschutz. 2016; 59:1544-1548. https://dx.doi. org/10.1007/s00103-016-2460-2
8. Rodríguez A, Sah R, Paniz A, Should the Holy Week 2020 be cancelled in Latin America due to the COVID-19 pandemic?. Travel Med Infect Dis. 2020; 36:101633. https:// dx.doi.org/10.1016/j.tmaid.2020.101633

9. Zhai $P$, Ding $Y$, Wu $X$, Long J, Zhong $Y$, The epidemiology, diagnosis and treatment of COVID-19. Int J Antimicrob Agents. 2020; 55(5):1-13. https://dx.doi.org/10.1016/j. ijantimicag. 2020.105955

10. Shigemura J, Ursano R, Morganstein J, Kurosawa M, Benedek D. Public responses to the novel 2019 coronavirus (2019-nCoV) in Japan: Mental health consequences and target populations. Psychiatry Clin Neurosci. 2020; 74(1):277-83 https:// dx.doi.org/10.1111/pcn.12988

11. La Republica [Internet]. Casos confirmados y muertos por Coronavirus en el Perú. Lima: La República; 2020 [citado el 20 de mayo del 2020]. Disponible en: https:// data.larepublica.pe/envivo/1552578-casosconfirmados-muertes-coronavirus-peru

12. Ministerio de Salud del Perú [internet]. Sala Situacional COVID-19. Lima: MINSA; 2020 [citado el 20 de mayo del 2020]. Disponible en: https://covid19.minsa.gob. pe/sala situacional.asp

13. Hernández R, Fernández C, Baptista P. Metodología de la Investigación. México: McGraw-Hill Interamericana; 2010.

14. Naresth M. Investigación de Mercado 4ta. edición México: Ed. Pearson Prentice Hall; 2014.

15. Bolivar E, Villanueva A. Validación y confiabilidad del Cuestionario AQ-27 de actitudes estigmatizadoras hacia pacientes con esquizofrenia en un Hospital General 2015. Rev Neuropsiquiatr. 2017; 80(3):165171. https://dx.doi.org/10.20453/rnp. v80i3.3153

16. Alhomoud F, Alhomoud F. Your Health Essential for Your Hajj: Muslim pilgrims' knowledge, attitudes and practices regarding Middle East respiratory syndrome coronavirus (MERS-CoV) during Hajj season. J Infect Chemother. 2017; 23(1):286-292. https:// dx.doi.org/10.1016/j.jiac.2017.01.006 
17. Jian J, Bai P, He W, Wu F, Liu XF, Han DM, Liu $S$, Yang J. Gender differences in patients with COVID-19: Focus on severity and mortality. Front Public Health. 2020; 8:152. https:// dx.doi.org/10.3389/fpubh.2020.00152

18. Albarraka A, Mohammedb R, Al Elayanc A, Al Fawaz F, Al Masryc M, Al Shammari M. Middle East Respiratory Syndrome (MERS): Comparing the knowledge, attitude and practices of different health care workers. J Infect Public Health. 2019; https://dx.doi. org/10.1016/j.jiph.2019.06.029

19. Chughtai A, Seale H, Dung T, Maher L, Nga $P$, MacIntyre $C$. Current practices and barriers to the use of facemasks and respirators among hospital-based health care workers in Vietnam. Am J Infect Control. 2014; 43(1):72-77. https://dx.doi.org/10.1016/j. ajic.2014.10.009
20. Roy D, Tripathya S, Kumar KS, Sharmaa N, Kumar VS, Kaushal V. Study of knowledge, attitude, anxiety \& perceived mental healthcare need in Indian population during COVID-19 pandemic. Asian J Psychiatr. 2020; 51:102083. https://dx.doi.org/10.1016/j. ajp. 2020.102083

21. Krishnakumar B, Rana. COVID 19 in INDIA: Strategies to combat from combination threat of life and livelihood. J Microbiol Immunol Infect. 2020; S1684-1182(20)30084-0. https://dx.doi. org/10.1016/j.jmii.2020.03.024 